\title{
DECISION FUNCTIONS AND CHARACTERIZATION OF THEIR PROPERTIES
}

\author{
BORBÁLA FAZEKAS
}

Mathematics subject classification (2000): 26B25, 90B50, 91B06, 93E24, 26E60, $26 \mathrm{E} 25$.

Key words and phrases: decision function, convexity, least squares method, monotonicity, continuity, power means, Gini means, quasi-arithmetic means.

\section{REFERENCES}

[1] E. F. Beckenbach, R. Bellman, Inequalities, Springer, Berlin, 1961.

[2] P. S. Bullen, D. S. Mitrinović AND P. M. VAsić, Means and Their Inequalities, Reidel, Dordrecht, 1988.

[3] Z. DARóczy, Über eine Klasse von Mittelwerten, Publ. Math. Debrecen, 19, (1972), 211-217 (1973).

[4] C. GINI, Di una formula compressiva delle medie, Metron, 13, (1938), 3-22.

[5] G. H. Hardy, J. E. Littlewood And G. Pólya, Inequalities, Cambridge University Press, Cambridge, 1934, (first edition), 1952 (second edition).

[6] O. HöLDER, Über einen Mittelwerthsatz, Nachr. Ges. Wiss. Göttingen (1889), 38-47.

[7] D. S. Mitrinović, Analytic Inequalities, Springer, Berlin-Heidelberg-New York, 1970.

[8] Zs. PÁLES, Characterization of quasideviation means, Acta Math. Acad. Sci. Hungar., 40, (3-4) (1982), 243-260.

[9] Zs. PÁLES, How to make fair decisions?, General Inequalities, 5 (Oberwolfach, 1986) (W. Walter, ed.), International Series of Numerical Mathematics, 80, Birkhäuser, Basel, 1987, 439-450.

[10] Zs. PÁLES, A Hahn-Banach theorem for separation of semigroups andits applications, Aequationes Math., 37, (2-3) (1989), 141-161.

[11] A. W. Roberts, D. E. Varberg, Convex Functions, Pure and Applied Mathematics, 57, Academic Press, New York-London, 1973.

[12] E. ZEIDLER, Nonlinear Functional Analysis and its Applications I., Springer, New York-BerlinHeidelberg, 1986. 